\title{
Josephson Supercurrent through the Topological Surface States of Strained Bulk HgTe
}

\author{
Jeroen B. Oostinga, ${ }^{1}$ Luis Maier, ${ }^{1}$ Peter Schüffelgen, ${ }^{1}$ Daniel Knott, ${ }^{1}$ Christopher Ames, ${ }^{1}$ Christoph Brüne, ${ }^{1}$ \\ Grigory Tkachov, ${ }^{2}$ Hartmut Buhmann, ${ }^{1}$ and Laurens W. Molenkamp ${ }^{1}$ \\ ${ }^{1}$ Physikalisches Institut (EP3), University of Würzburg, Am Hubland, D-97074 Würzburg, Germany \\ ${ }^{2}$ Institut für Theoretische Physik und Astrophysik (TP4), University of Würzburg, Am Hubland, D-97074 Würzburg, Germany
}

(Received 25 January 2013; revised manuscript received 4 April 2013; published 28 May 2013)

\begin{abstract}
Strained bulk HgTe is a three-dimensional topological insulator, whose surface electrons have a high mobility ( $\sim 30000 \mathrm{~cm}^{2} / \mathrm{Vs}$ ), while its bulk is effectively free of mobile charge carriers. These properties enable a study of transport through its unconventional surface states without being hindered by a parallel bulk conductance. Here, we show transport experiments on $\mathrm{HgTe}$-based Josephson junctions to investigate the appearance of the predicted Majorana states at the interface between a topological insulator and a superconductor. Interestingly, we observe a dissipationless supercurrent flow through the topological surface states of $\mathrm{HgTe}$. The current-voltage characteristics are hysteretic at temperatures below $1 \mathrm{~K}$, with critical supercurrents of several microamperes. Moreover, we observe a magnetic-field-induced Fraunhofer pattern of the critical supercurrent, indicating a dominant $2 \pi$-periodic Josephson effect in the unconventional surface states. Our results show that strained bulk $\mathrm{HgTe}$ is a promising material system to get a better understanding of the Josephson effect in topological surface states, and to search for the manifestation of zero-energy Majorana states in transport experiments.
\end{abstract}

DOI: 10.1103/PhysRevX.3.021007 Subject Areas: Mesoscopics, Superconductivity, Topological Insulators

A strained, undoped layer of HgTe thicker than approximately $50 \mathrm{~nm}$ is a three-dimensional topological insulator. While $\mathrm{HgTe}$ does not exhibit an intrinsic band gap in its bulk states, a strain-induced band gap is opened [1,2] when a HgTe layer is epitaxially grown on a CdTe substrate. Owing to the topological properties of $\mathrm{HgTe}$, a single family of gapless Dirac states appears at each of the twodimensional surfaces [3]. Transport measurements [4] and terahertz experiments $[5,6]$ have indeed confirmed that two-dimensional Dirac states exist at the surface of strained bulk HgTe. More recently, transport experiments on Josephson junctions based on these HgTe layers have demonstrated that superconductivity can be induced in the topological surface states by proximity to a superconductor [7]. Since Majorana fermions-particles that are indistinguishable from their own antiparticles-are expected to emerge at the interface between a superconductor and a topological insulator [8], superconducting weak links of strained bulk $\mathrm{HgTe}$ are promising solid-state systems to investigate the manifestation of such Majorana quasiparticle excitations in transport experiments [9].

Josephson junctions are electronic devices consisting of two superconductors connected by a weak link. Owing to the coupling between both superconducting condensates, Cooper pairs can be transferred dissipationless from one superconductor to the other. This is the Josephson effect, and it gives rise to a supercurrent through a weak link,

Published by the American Physical Society under the terms of the Creative Commons Attribution 3.0 License. Further distribution of this work must maintain attribution to the author(s) and the published article's title, journal citation, and DOI. which has been studied extensively in a large variety of material systems, from thin insulating layers to conducting films, semiconducting nanowires, carbon nanotubes, and graphene [10-15]. When a conducting material is sandwiched between two superconductors, the proximity effect leads to induced superconductivity in the normal conductor, and electronic transport is mediated by Andreev bound states (which are phase-coherent superpositions of electron and hole excitations) $[12,16]$. It has been predicted that a topological-insulator weak link supports nonchiral, zeroenergy Andreev modes in the surface states (when the phase difference between both superconductors is $\phi=\pi$ or odd multiples of $\pi$ ) [8]. These zero-energy modes are a consequence of the induced $p$-like pairing symmetry of the topological surface states, and they are intimately related to Majorana fermions, which give rise to a $4 \pi$-periodic Josephson effect $[9,17]$.

Recent experiments have shown the first observation of a Josephson supercurrent through topological insulators $\mathrm{Bi}_{2} \mathrm{Se}_{3}$ [18,19] and $\mathrm{Bi}_{2} \mathrm{Te}_{3}$ [20,21]. However, these Bi-based materials exhibit a residual bulk conductance that dominates electronic transport and obstructs an unambiguous investigation of induced superconductivity in the topological surface states. Contrary to the Bi-based topological insulators, the bulk of strained $\mathrm{HgTe}$ is effectively insulating [4], enabling the exploration of the Josephson effect in its topological surface states [7]. For this reason, we have fabricated lateral HgTe-based Josephson junctions (with closely spaced superconducting $\mathrm{Nb}$ electrodes on the top surface [22]) to study the occurrence of Andreev bound states at the surface, and to investigate superconducting transport through these unconventional states [Fig. 1(a)]. 
(a)

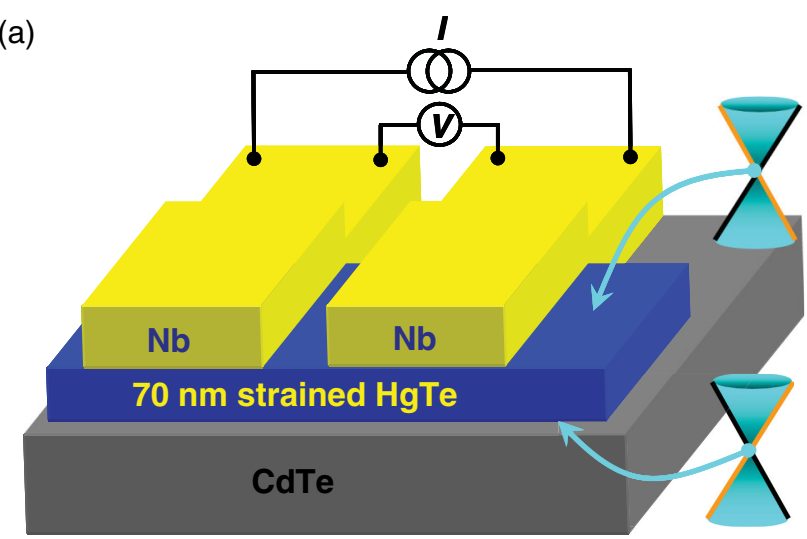

(b)

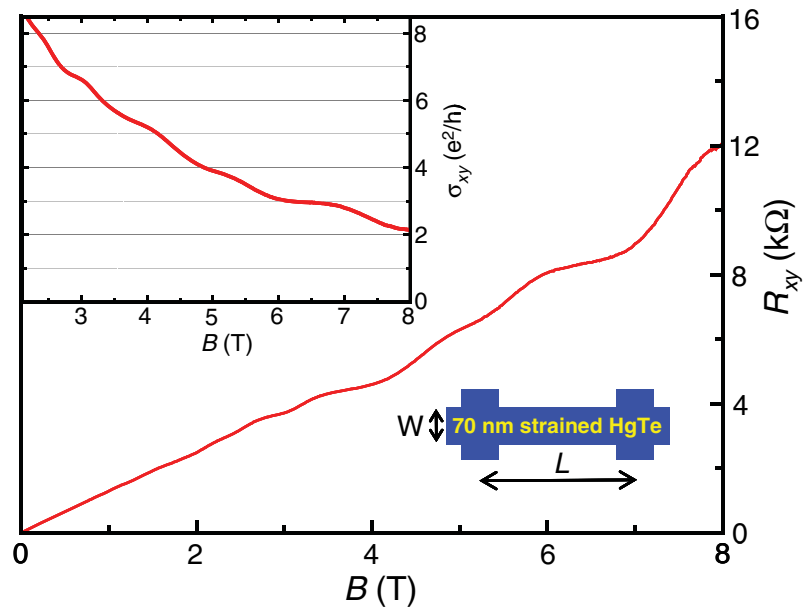

FIG. 1. (a) Schematic picture of a lateral Josephson junction based on a strained, undoped HgTe layer of $70 \mathrm{~nm}$ thickness. The Dirac states residing at the surfaces of the HgTe layer form the weak link between the $\mathrm{Nb}$ electrodes. Transport measurements on such a junction are performed in current-bias mode while measuring the voltage across the junction to investigate superconducting transport through the surface states. (b) Transport measurements at $T \approx 70 \mathrm{mK}$ on a Hall bar device with $L=$ $600 \mu \mathrm{m}$ and $W=200 \mu \mathrm{m}$, based on the same HgTe layer as the Josephson junction. The magnetic-field dependence of the Hall resistance $R_{x y}$ shows the development of plateaus, implying transport through two-dimensional states. The inset shows the quantization of the Hall conductivity $\sigma_{x y}$ at odd as well as even Landau level filling factors, characteristic of Dirac states at top and bottom surfaces with slightly different densities [4]. This observation demonstrates that the bulk conductance is negligible, and transport is effectively through the topological surface states.

First we identify the origin of electronic transport in the 70-nm-thick strained HgTe layer that we used as a weak link. For this purpose, we have fabricated, from the same wafer as the superconducting devices described below, a six-terminal Hall bar device with a channel length of $600 \mu \mathrm{m}$ and width of $200 \mu \mathrm{m}$ (same geometry as the device in Ref. [4]), and measured the magnetic-field dependence of its Hall resistance at cryogenic temperatures [Fig. 1(b)]. From the low-field data, the mobility and charge density of the conduction electrons are extracted: $\mu \approx 26000 \mathrm{~cm}^{2} / \mathrm{Vs}$ and $n \approx 5.5 \times 10^{11} \mathrm{~cm}^{-2}$. Interestingly, Fig. 1(b) shows a series of quantization plateaus of the Hall resistance when the magnetic field is increased above $B \approx 2 \mathrm{~T}$. This observation provides evidence that transport is effectively through two-dimensional states. The magnetic-field dependence of the Hall conductivity [inset of Fig. 1(b)] reveals the development of plateaus at $\sigma_{x y}=$ $\frac{2 e^{2}}{h}, \frac{3 e^{2}}{h}, \frac{4 e^{2}}{h}$ and weak plateaulike features close to $\sigma_{x y}=\frac{5 e^{2}}{h}$ and $\sigma_{x y}=\frac{7 e^{2}}{h}$ (comparable to the reported observations in Ref. [4]). The appearance of quantum Hall plateaus corresponding to even as well as odd filling factors, with the odd ones being most robust at low magnetic fields, is characteristic of a single family of Dirac states at the top as well as the bottom surface with slightly different densities. This implies that the bulk is effectively free of mobile carriers, and electronic transport is predominantly through the twodimensional surface states of strained $\mathrm{HgTe}$ [4-7].

Having identified that electronic transport occurs effectively through Dirac surface states, we can now investigate transport through a lateral Josephson junction and study the nature of induced superconductivity in the topological surface states [Fig. 2(a); this junction has been fabricated from the same HgTe layer by using two $\mathrm{Nb}$ contacts at the top surface as superconducting electrodes [22]]. We measured the differential resistance $d V / d I$ of the junction as a function of current bias, temperature, and magnetic field in a measurement setup equipped with appropriate low-pass filters for supercurrent measurements [23]. In order to have the $\mathrm{Nb}$ electrodes in the superconducting state, the measurements were all done at temperatures well below the critical temperature of $\mathrm{Nb}\left(T_{c} \approx 9 \mathrm{~K}\right)$. Our measurements at the base temperature $(T=25 \mathrm{mK})$ of the dilution refrigerator clearly show two transport regimes [Fig. 2(b)]: A dissipationless supercurrent appears in the junction when the bias is lower than a critical value $I_{c}$ (i.e., $R=0$ if $I<I_{c}$ ), while a dissipating current occurs at higher current bias (with $R_{n} \approx 50 \Omega$ ).

Let us inspect the resistive regime in more detail. When $d V / d I$ is plotted versus the measured voltage across the junction [Fig. 2(c)], we observe that the differential resistance slightly decreases when the voltage is below $V=$ $2 \Delta / e \approx 2 \mathrm{mV}$ (with $\Delta \approx 1 \mathrm{meV}$ the superconducting gap of $\mathrm{Nb}$ ), indicating that Andreev reflections occur at both $\mathrm{HgTe}-\mathrm{Nb}$ interfaces, and that these interfaces are relatively transparent [24]. Moreover, a clear feature is present at $V=\Delta / e \approx 1 \mathrm{mV}$, which can be attributed to multiple Andreev reflections [24]. The subharmonic gap structure related to higher-order Andreev reflections is missing, probably because of smearing of the population of Andreev bound states in the course of multiple passages across a voltage-biased junction [25]. The smearing of the distribution of Andreev levels may be viewed as a nonequilibrium self-heating effect, as suggested previously for InAs-based superconducting junctions [26]. The inset of Fig. 2(c) shows that reproducible, aperiodic fluctuations 
(a)

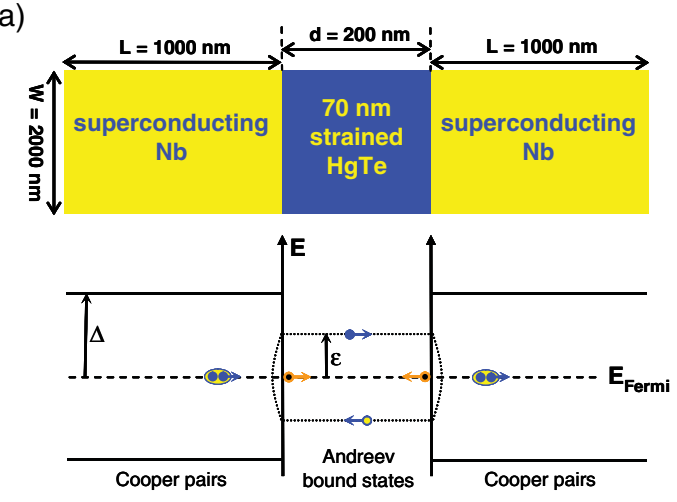

(c)

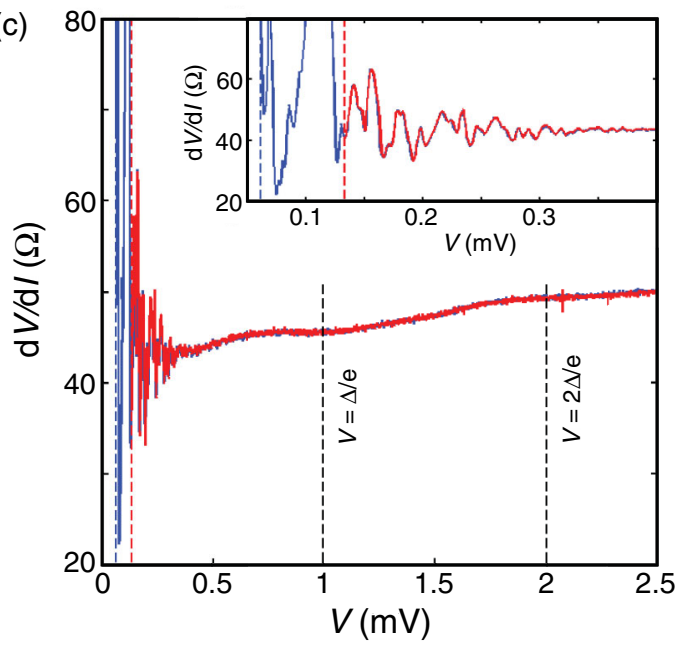

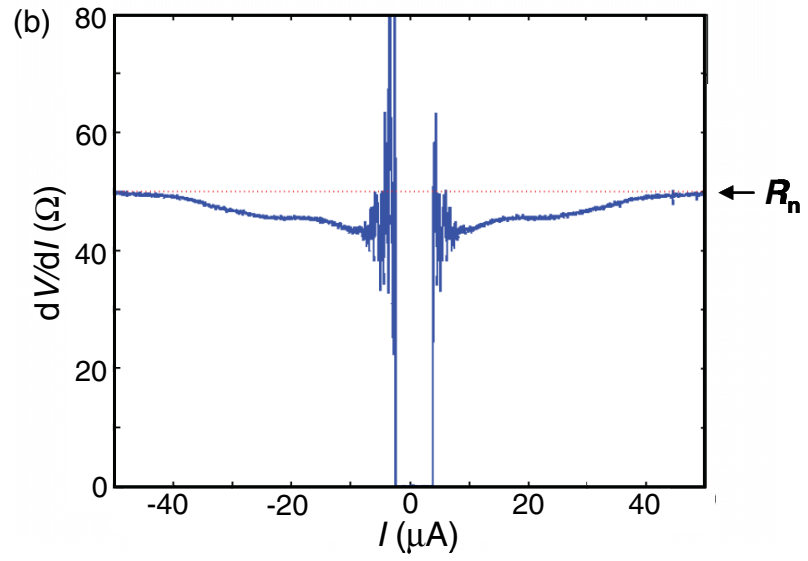

(d)

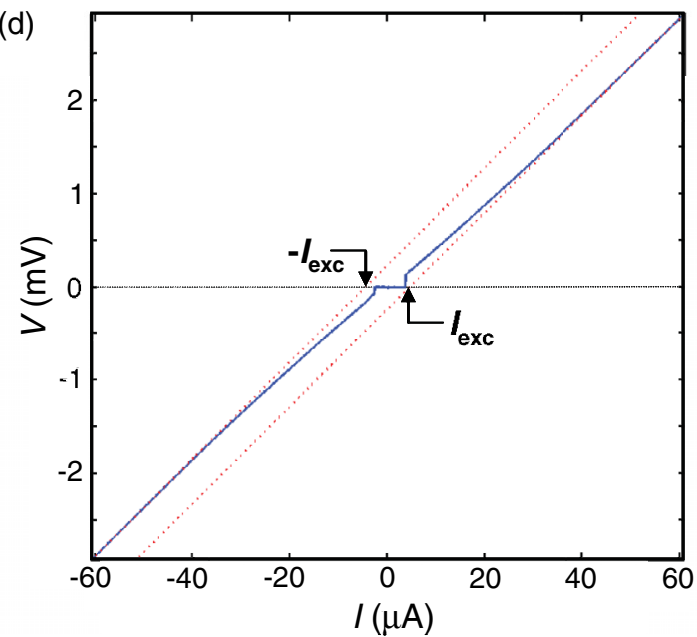

FIG. 2. (a) Geometric dimensions of the Josephson junction and the corresponding energy diagram showing Andreev modes at finiteenergy $(\varepsilon>0)$ and zero-energy values $(\varepsilon=0)$. The presence of zero-energy modes is predicted to give rise to a $4 \pi$-periodic supercurrent through the topological surface states [8]. However, normal scattering in the weak link can remove unprotected zero modes, leading to a $2 \pi$-periodic Josephson effect $[9,17,35]$. (b) Differential resistance versus current bias at $T=25 \mathrm{mK}$ (ascending bias sweep). In the high-current regime, the differential resistance converges to the value of the normal resistance $R_{n} \approx 50 \Omega$. When the current is decreased below a critical value, the differential resistance drops to zero, corresponding to a supercurrent regime. (c) Differential resistance plotted versus the voltage across the junction at $T=25 \mathrm{mK}$. When $V<2 \Delta / \mathrm{e}, d V / d I$ decreases because of Andreev reflections. The feature at $V \approx \Delta / \mathrm{e}$ can be attributed to multiple Andreev reflections. The inset shows reproducible, aperiodic fluctuations, which are related to the Josephson effect. The red (blue) curve is a bias sweep in the ascending (descending) direction. The red and blue dashed, vertical lines indicate the switching $\left(V_{s}\right)$ and retrapping voltages $\left(V_{r}\right)$, respectively. (d) Nonlinear $I$ - $V$ characteristic of the junction at $T=25 \mathrm{mK}$ (ascending bias sweep). The red dotted lines, with a slope corresponding to $R_{n} \approx 50 \Omega$, cross the $V=0$ axis at a finite current value. This is the excess current $I_{\text {exc }} \approx 4.5 \mu \mathrm{A}$, which is directly related to Andreev reflections in the junction $[24,25,27,28]$.

are observable at $V<0.4 \mathrm{mV}$ (where the current bias is just above the critical current). These fluctuations originate from the Josephson effect and will be discussed in more detail when we consider the magnetic-field-dependent measurements. The occurrence of multiple Andreev reflections at the $\mathrm{HgTe}-\mathrm{Nb}$ interfaces gives rise to nonlinear current-voltage $(I-V)$ characteristics in the subgap regime [Fig. 2(d); $V<2 \Delta / e$ ]. Even for $V>2 \Delta / e$, Andreev reflection processes contribute to the total current through the junction [27]. This results in an excess current $\left(I_{\mathrm{exc}}\right)$, which is usually of the order of the critical current and can be determined from the measurements of Fig. 2(d):
$I_{\text {exc }}=I(V)-V / R_{n} \approx 4.5 \mu \mathrm{A}$ [where $R_{n} \approx 50 \Omega$ is the differential resistance and $I(V)$ is the measured current at $V>2 \Delta / e$; see red dotted lines in Figs. 2(b) and 2(d)]. Based on Blonder-Tinkham-Klapwijk theory $[24,28]$ and using the values of $I_{\text {exc }}$ and $R_{n}$, the transparency of the $\mathrm{HgTe}-\mathrm{Nb}$ interfaces can be estimated, yielding $T_{\text {int }} \approx 0.5$ [29]. This indicates that the interfaces have a moderate transparency [11].

When we inspect the supercurrent regime of the junction, we clearly observe hysteresis in the measured $I-V$ characteristics [Fig. 3(a)]. The critical current corresponding to the transition from supercurrent to resistive regime 

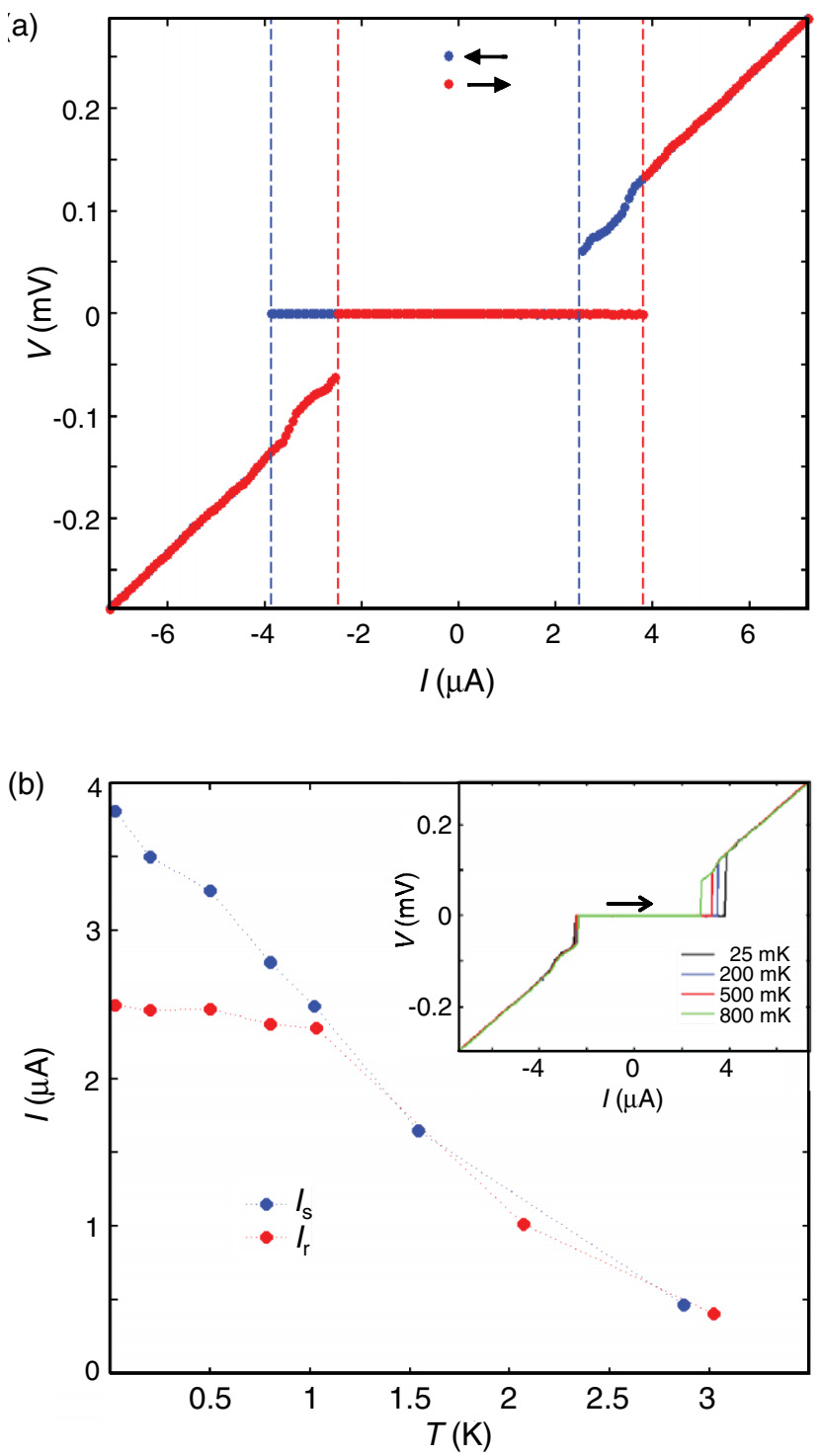

FIG. 3. (a) Ascending (red) and descending (blue) $I$ - $V$ characteristics, showing pronounced hysteresis $(T=25 \mathrm{mK})$. The switching current, which is the critical current of the transition to the normal-state regime, is larger than the retrapping current, which is the critical current of the transition to the supercurrent regime. (b) The temperature dependence of the switching and retrapping current $\left(I_{s}\right.$ and $\left.I_{r}\right)$, showing that hysteresis is absent at $T>1 \mathrm{~K}$. Note that the supercurrent regime disappears at $T>4 \mathrm{~K}$. The inset shows hysteretic $I-V$ characteristics in the temperature range of $25-800 \mathrm{mK}$.

is larger than the critical current corresponding to the transition from resistive to supercurrent regime-i.e., at $T=25 \mathrm{mK}$, the switching and retrapping current are $I_{s} \approx 3.8 \mu \mathrm{A}$ and $I_{r} \approx 2.5 \mu \mathrm{A}$, respectively. The temperature dependence of the $I-V$ characteristics shows that hysteresis is only present in the temperature range up to $1 \mathrm{~K}$, whereas $I_{s}$ and $I_{r}$ exhibit approximately equal values at higher temperatures [Fig. 3(b)]. Switching and retrapping current decrease with increasing temperature, until the supercurrent regime disappears at $T>4 \mathrm{~K}$. Hysteresis usually occurs in underdamped Josephson junctions, i.e., in junctions that are effectively shunted by a large resistance and capacitance $[10,11]$. However, the capacitance of our lateral junction is very small $\left(C \approx \varepsilon_{0} t_{\mathrm{SC}} W_{\mathrm{SC}} / d \approx 34 \mathrm{aF}\right.$, where $\varepsilon_{0}$ is the vacuum permittivity, $t_{\mathrm{SC}} \approx 90 \mathrm{~nm}$ is the thickness of the superconducting electrodes, and $W_{\mathrm{SC}} \approx$ $8.5 \mu \mathrm{m}$ is the total width of the pair of parallel electrodes), and the Stewart-McCumber parameter of the resistively and capacitively shunted junction model is only $\beta_{c}=$ $2 e I_{c} R_{n}^{2} C / \hbar \approx 0.001$ [11]. Since $\beta_{c} \ll 1$, the junction is overdamped, and no hysteresis is expected in the $I-V$ characteristics [11]. Alternatively, self-heating effects are known to lead to hysteresis in Josephson junctions with large critical currents $[10,30]$. In our junction, the power density at the retrapping point is estimated to be $P / V_{S S} \approx$ $I_{r} V_{r} / V_{S S} \sim 10 \mathrm{nW} / \mu \mathrm{m}^{3}$ (where $V_{S S} \sim 0.01 \mu \mathrm{m}^{3}$ is an estimation of the volume corresponding to the surface states through which the current flows). This value indeed suggests that the observed hysteresis may well be due to electron heating (since it is consistent with Ref. [30]).

The $I_{c} R_{n}$ product-i.e., the critical current multiplied by the normal state resistance-is a characteristic junction parameter that provides useful information about superconducting transport through the Josephson junction. The $I_{c} R_{n}$ product is usually around $\Delta / e$ for short junctions (i.e., junctions with a Thouless energy $E_{\mathrm{Th}}$ larger than the superconducting gap, $\Delta<E_{\mathrm{Th}}=\hbar D / L^{2}$, where $D$ is the diffusion constant and $L$ is the junction length), while it is much smaller for long junctions (i.e., junctions with $E_{\mathrm{Th}}<\Delta$ ) [31]. For our junction, we obtain $I_{c} R_{n} \approx 0.2 \mathrm{mV}$ at the lowest temperature, which is about 5 times smaller than $\Delta / e \approx 1 \mathrm{mV}$. Possibly, this indicates that the junction is in the long junction limit, where the superconducting coherence length $\xi$ is smaller than the spacing $d$ between both $\mathrm{Nb}$ electrodes (i.e., $\xi<d \approx 200 \mathrm{~nm}$ ). Alternatively, the suppressed $I_{c} R_{n}$ product may be an indication of insufficient interface transparency [32].

The measurements discussed so far do not identify a contribution from zero-energy Majorana bound states to the supercurrent. Contrary to Andreev bound states in conventional materials, which give rise to a $2 \pi$-periodic Josephson effect $\left(I_{c} \propto \sin \phi\right)$, the presence of zero-energy Majorana states in topological insulators yields a $4 \pi$-periodic Josephson effect $\left[I_{c} \propto \sin (\phi / 2)\right][8,9]$. It is therefore beneficial to study the current-phase relation to identify a contribution from Majorana states to the supercurrent. The critical supercurrent is maximal at zero field and decreases with an increasing magnetic field in an oscillatory way, yielding a Fraunhofer diffraction pattern if the supercurrent flows uniformly through the junction [11]. The periodicity is $\Delta B=\Phi_{0} / A$ for a $2 \pi$-periodic supercurrent (where $\Phi_{0}=h / 2 e$ is the flux quantum, and $A$ is the junction area). However, it is predicted that the periodicity is twice as large for a $4 \pi$-periodic supercurrent [9], i.e., $\Delta B=2 \Phi_{0} / A$. 
Thus, exploring the magnetic-field dependence of the critical current of our junction is expected to be an effective method to identify the nature of the supercurrent-carrying states.

When we measure $I-V$ characteristics as a function of an applied perpendicular magnetic field, we clearly observe a Fraunhofer-like diffraction pattern of the critical current, with a periodicity of $\Delta B \approx 1.1 \mathrm{mT}$ [Fig. 4(a)]. The pattern deviates from a perfect Fraunhofer pattern, and it indicates that the supercurrent is not fully uniform (note that measurements on different junctions have shown that this deviation is indeed sample specific) [11]. To determine the magnetic flux through the junction, we need to consider the effective junction area, which is larger than the area between the electrodes, because the penetration length of the niobium films in a perpendicular magnetic field is approximately $\lambda \approx 350 \mathrm{~nm}$ [7]. Thus, the periodicity of the Fraunhofer pattern corresponds very well to $\Delta B \approx \Phi_{0} / A$, implying a dominating $2 \pi$-periodic supercurrent through the topological surface states [33]. Note that we do not expect Josephson screening currents to affect our measurements because the junction is in the narrow junction regime [i.e., the junction width is smaller than the Josephson penetration depth: $W<\lambda_{J}=\sqrt{\Phi_{0} t_{\mathrm{HgTe}} W / 2 \pi \mu_{0} I_{c}(d+2 \lambda)} \approx 3.3 \mu \mathrm{m}$, where $\mu_{0}$ is the vacuum permeability and $t_{\mathrm{HgTe}}$ is the thickness of the HgTe layer]. Also, flux-focusing effects are not expected to play a role in our junction because the planar dimensions of the $\mathrm{Nb}$ electrodes are larger than $\lambda \approx$ $350 \mathrm{~nm}$ (i.e., the $\mathrm{Nb}$ electrodes are in the wide-electrode limit [34]).

Besides a Fraunhofer pattern, Fig. 4(a) shows distinct features in the resistive regime at currents just above the critical current. These features are most pronounced above the main lobe of the Fraunhofer pattern but are also clearly visible above the first few side lobes. These features correspond to the $d V / d I$ fluctuations that we already observed in Fig. 2(c) at small biases $(V<0.4 \mathrm{mV})$. To investigate the origin of these fluctuations, we have measured $d V / d I$ versus $V$ at different magnetic-field values ranging from $B=0$ to $1.12 \mathrm{mT}$ [Fig. 4(b)]. These data show that the fluctuations are maximal at zero field and decrease with an increasing magnetic field. At $B \approx 1.1 \mathrm{mT}$, when one flux quantum is captured in the junction $\left(B=\Phi_{0} / A\right)$, the fluctuations vanish completely. Moreover, Fig. 4(a) shows that the fluctuations appear and disappear with the same periodicity as the Fraunhofer pattern. Obviously, these $d V / d I$ fluctuations are a manifestation of the Josephson effect in the resistive regime [10]. However, the exact origin of these fluctuations, which we observed in all $\mathrm{HgTe}$ junctions studied so far, remains somewhat obscure.

In conclusion, our experimental data show that the supercurrent through the topological surface states of HgTe exhibits no clear signature of Majorana bound states. In contrast to some recent claims in the literature [19], this
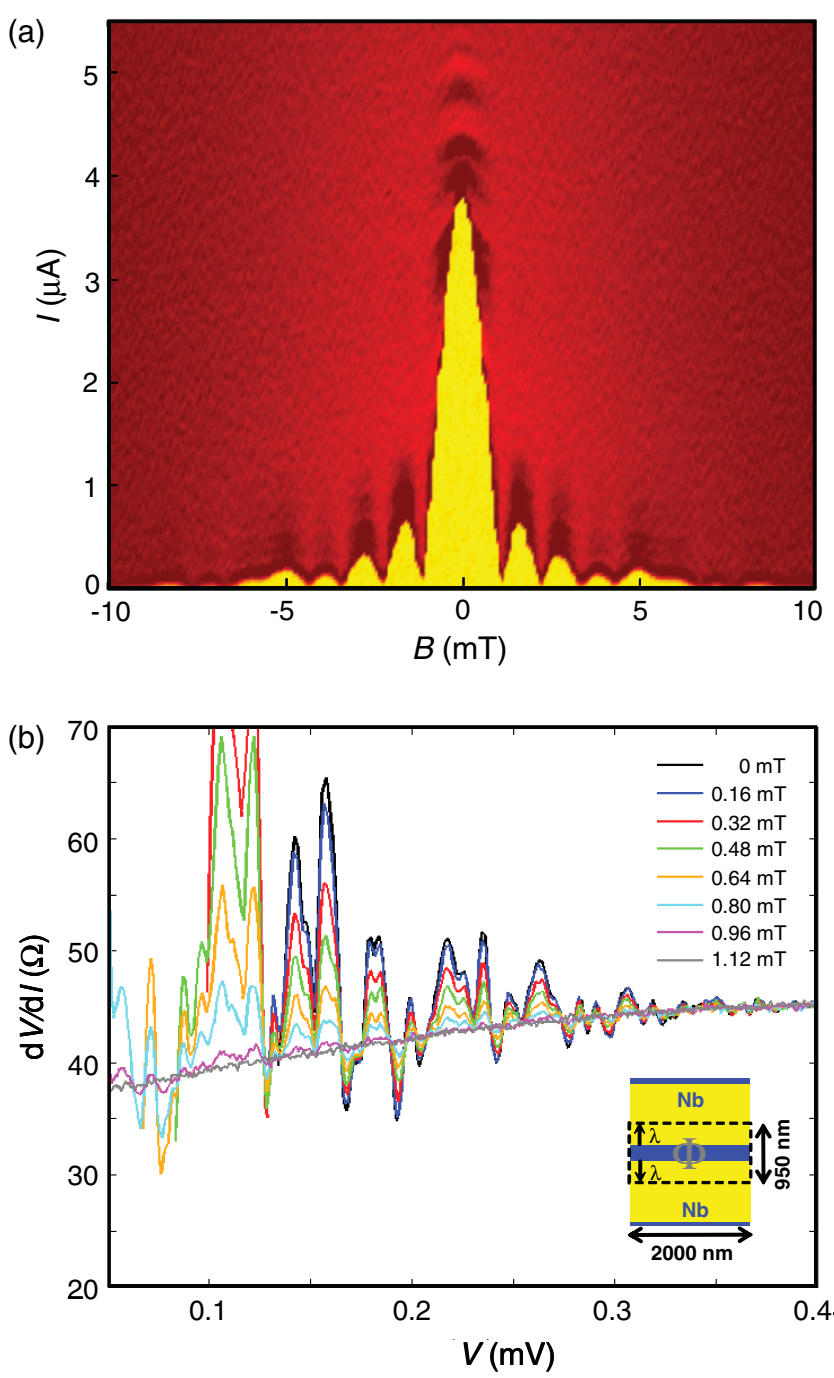

FIG. 4. (a) Color plot of $d V / d I$ data versus current bias (ascending sweeps) and an applied magnetic field $(T=25 \mathrm{mK})$. The $d V / d I$ data are numerically derived from measured $I-V$ characteristics at different magnetic-field values. The yellow color corresponds to the supercurrent regime $(d V / d I=0)$ and the red color to the normal-state regime $(d V / d I>0)$. A Fraunhofer pattern appears with a periodicity of $\Delta B \approx 1.1 \mathrm{mT}\left(\approx \Phi_{0} / A\right)$, providing evidence that the supercurrent is carried predominantly by $2 \pi$-periodic Andreev bound states. The deviation from a perfect Fraunhofer pattern indicates a nonuniform supercurrent through the junction. (b) Measured $d V / d I$ versus the voltage across the junction (ascending bias sweeps) at different magnetic-field values $(T=25 \mathrm{mK})$. The amplitude of the reproducible, aperiodic fluctuations is maximal at $B=0$, decreases with the magnetic field, and vanishes completely at $B \approx 1.1 \mathrm{mT}$ (when one flux quantum is captured in the effective junction area). This implies that the fluctuations originate from the Josephson effect. The effective junction length of $950 \mathrm{~nm}$ incorporates the magnetic penetration length of the $\mathrm{Nb}$ (see inset).

should not come as a surprise. Actually, theory expects that quasiparticle scattering in the junction removes unprotected, zero-energy modes, leaving an energy gap in the Andreev bound states-similar to conventional junctions 
[12,35]. Effectively, the $4 \pi$ periodicity of the unprotected Andreev bound states turns into a $2 \pi$ periodicity when the spectrum is gapped. Only the zero-energy Andreev bound states with the momentum perpendicular to the interface are topologically protected and remain ungapped, but since this number of modes is predicted to be orders of magnitude smaller than the number of unprotected modes $[9,17]$, a $2 \pi$-periodic Josephson effect may dominate the supercurrent-as is the case in our experiments. Moreover, the emergence of zero-energy Majorana states depends on the properties of the interface, such as the transparency and the position of the Fermi level with respect to the Dirac point of the topological states at the interface [36,37]. Although we do not observe a clear sign of the presence of protected Majorana bound states, our results show that strained bulk HgTe is a promising material system to get a better understanding of the Josephson effect in topological surface states, and to search for the manifestation of zeroenergy Majorana states in transport experiments.

We thank E. M. Hankiewicz, B. Trauzettel, and P. Brouwer for useful discussions. This work was financially supported by the German Research Foundation (DFG-JST joint research program "Topological Electronics" and Grant No. TK 60/1-1), the EU ERC-AG program (project 3-TOP), and the DARPA program.

[1] L. Fu and C.L. Kane, Topological Insulators with Inversion Symmetry, Phys. Rev. B 76, 045302 (2007).

[2] X. Dai, T. L. Hughes, X.-L. Qi, Z. Fang, and S.-C. Zhang, Helical Edge and Surface States in HgTe Quantum Wells and Bulk Insulators, Phys. Rev. B 77, 125319 (2008).

[3] L. Fu, C. L. Kane, and E. J. Mele, Topological Insulators in Three Dimensions, Phys. Rev. Lett. 98, 106803 (2007).

[4] C. Brüne, C. X. Liu, E. G. Novik, E. M. Hankiewicz, H. Buhmann, Y. L. Qi, Z.X. Shen, S.C. Zhang, and L. W. Molenkamp, Quantum Hall Effect from the Topological Surface States of Strained Bulk HgTe, Phys. Rev. Lett. 106, 126803 (2011).

[5] J. N. Hancock, J. L. M. van Mechelen, A. B. Kuzmenko, D. van der Marel, C. Brüne, E. G. Novik, G. V. Astakhov, H. Buhmann, and L. W. Molenkamp, Surface State Charge Dynamics of a High-Mobility Three-Dimensional Topological Insulator, Phys. Rev. Lett. 107, 136803 (2011).

[6] A. M. Shuvaev, G. V. Astakhov, G. Tkachov, C. Brüne, H. Buhmann, L. W. Molenkamp, and A. Pimenov, Terahertz. quantum Hall effect of Dirac fermions in a topological insulator, Phys. Rev. B 87, 121104 (2013).

[7] L. Maier, J. B. Oostinga, D. Knott, C. Brüne, P. Virtanen, G. Tkachov, E. M. Hankiewicz, C. Gould, H. Buhmann, and L. W. Molenkamp, Induced Superconductivity in the Three-Dimensional Topological Insulator HgTe, Phys. Rev. Lett. 109, 186806 (2012).

[8] L. Fu and C. L. Kane, Superconducting Proximity Effect and Majorana Fermions at the Surface of a Topological Insulator, Phys. Rev. Lett. 100, 096407 (2008).
[9] C. W. J. Beenakker, Search for Majorana Fermions in Superconductors, Annu. Rev. Condens. Matter Phys. 4, 113 (2013).

[10] K. K. Likharev, Superconducting Weak Links, Rev. Mod. Phys. 51, 101 (1979).

[11] M. Tinkham, Introduction to Superconductivity (McGrawHill, New York, 1996).

[12] T. M. Klapwijk, Proximity Effect from an Andreev Perspective, J. Supercond. 17, 593 (2004).

[13] Y.-J. Doh, J. A. van Dam, A. L. Roest, E. P. A. M. Bakkers, L.P. Kouwenhoven, and S. De Franceschi, Tunable Supercurrent through Semiconductor Nanowires, Science 309, 272 (2005).

[14] P. Jarillo-Herrero, J. A. van Dam, and L. P. Kouwenhoven, Quantum Supercurrent Transistors in Carbon Nanotubes, Nature (London) 439, 953 (2006).

[15] H. B. Heersche, P. Jarillo-Herrero, J. B. Oostinga, L. M. K. Vandersypen, and A.F. Morpurgo, Bipolar Supercurrent in Graphene, Nature (London) 446, 56 (2007).

[16] A. A. Golubov, M. Y. Kupriyanov, and E. Il'ichev, The Current-Phase Relation in Josephson Junctions, Rev. Mod. Phys. 76, 411 (2004).

[17] G. Tkachov and E. M. Hankiewicz, Spin-Helical Transport in Normal and Superconducting Topological Insulators, Phys. Status Solidi B 250, 215 (2013).

[18] B. Sacépé, J. B. Oostinga, J. Li, A. Ubaldini, N. J. G. Couto, E. Giannini, and A.F. Morpurgo, Gate-Tuned Normal and Superconducting Transport at the Surface of a Topological Insulator, Nat. Commun. 2, 575 (2011).

[19] J. R. Williams, A. J. Bestwick, P. Callagher, S. S. Hong, Y. Cui, A.S. Bleich, J.G. Analytis, I. R. Fisher, and D. Goldhaber-Gordon, Unconventional Josephson Effect in Hybrid Superconductor-Topological Insulator Devices, Phys. Rev. Lett. 109, 056803 (2012).

[20] M. Veldhorst, M. Snelder, M. Hoek, T. Gang, V. K. Guduru, X. L. Wang, U. Zeitler, W. G. van der Wiel, A. A. Golubov, H. Hilgenkamp, and A. Brinkman, Josephson Supercurrent through a Topological Insulator Surface State, Nat. Mater. 11, 417 (2012).

[21] F. Qu, F. Yang, J. Shen, Y. Ding, J. Chen, Z. Ji, G. Liu, J. Fan, X. Jing, C. Yang, and L. Lu, Strong Superconducting Proximity Effect in $\mathrm{Pb}-\mathrm{Bi}_{2} \mathrm{Te}_{3}$ Hybrid Structures, Sci. Rep. 2, 339 (2012).

[22] An HgTe layer of $70 \mathrm{~nm}$ thickness is grown on a CdTe substrate by molecular beam epitaxy. The HgTe layer is etched in an Ar plasma to get a 2- $\mu \mathrm{m}$-wide HgTe stripe. On the top surface of the HgTe stripe, two 70-nm-thick $\mathrm{Nb}$ electrodes-with a spacing of $200 \mathrm{~nm}$ - are deposited by using e-beam lithography, ultrahigh vacuum sputtering, and liftoff techniques. Additionally, a protective bilayer consisting of $10 \mathrm{~nm} \mathrm{Al}$ and $10 \mathrm{~nm} \mathrm{Ru}$ is deposited on top of the $\mathrm{Nb}$ electrodes. Note that, prior to $\mathrm{Nb}$ sputtering, the $\mathrm{HgTe}$ surface is cleaned by exposing it to a very mild, low-power Ar plasma, resulting in more transparent $\mathrm{HgTe}-\mathrm{Nb}$ interfaces without affecting the quality of the surface [7].

[23] The transport measurements are performed in current-bias mode in an Oxford dilution refrigerator at a base temperature of $25 \mathrm{mK}$. The differential resistance is measured as a function of current bias, temperature, and magnetic field by using standard lock-in detection techniques, and the dc voltage across the junction is measured simultaneously 
(note that the data of Figs. 3(a), 3(b), and 4(a) are obtained by pure dc measurements). The signals of all four wires to and from the junction are filtered at different temperature stages: low-pass filters at room temperature; radiofrequency "eccosorb" filters at $4 \mathrm{~K}$; low-pass RC filters and $\mathrm{Cu}$ powder filters at $25 \mathrm{mK}$. Filtering is required to suppress the high-frequency noise, which is destructive for a Josephson supercurrent [13-15,18].

[24] G. E. Blonder, M. Tinkham, and T.M. Klapwijk, Transition from Metallic to Tunneling Regimes in Superconducting Microconstrictions: Excess Current, Charge Imbalance, and Supercurrent Conversion, Phys. Rev. B 25, 4515 (1982).

[25] K. Flensberg and J. B. Hansen, Subharmonic Energy-Gap Structure and Heating Effects in Superconducting Niobium Point Contacts, Phys. Rev. B 40, 8693 (1989).

[26] J. P. Heida, B. J. van Wees, T. M. Klapwijk, and G. Borghs, Critical Currents in Ballistic Two-Dimensional InAsBased Superconducting Weak Links, Phys. Rev. B 60, 13135 (1999).

[27] T. M. Klapwijk, G.E. Blonder, and M. Tinkham, Explanation of Subharmonic Energy Gap Structure in Superconducting Contacts, Physica B+C 109-110, 1657 (1982).

[28] K. Flensberg, J. B. Hansen, and M. Octavio, Subharmonic Energy-Gap Structure in Superconducting Weak Links, Phys. Rev. B 38, 8707 (1988).

[29] This value is the average transparency over all transport channels and is estimated by using the general expression for the excess current in one-dimensional superconductor-normal-metal-superconductor junctions, multiplied by the number of transport channels in the junction $[24,28]$.

[30] H. Courtois, M. Meschke, J. T. Peltonen, and J. P. Pekola, Origin of Hysteresis in a Proximity Josephson Junction, Phys. Rev. Lett. 101, 067002 (2008).

[31] P. Dubos, H. Courtois, B. Pannetier, F. K. Wilhelm, A. D. Zaikin, and G. Schön, Josephson Critical Current in a Long Mesoscopic S-N-S Junction, Phys. Rev. B 63, 064502 (2001).

[32] A. Chrestin and U. Merkt, High Characteristic Voltages in $\mathrm{Nb} /$ p-type InAs/Nb Josephson Junctions, Appl. Phys. Lett. 70, 3149 (1997).

[33] See Supplemental Material at http://link.aps.org/ supplemental/10.1103/PhysRevX.3.021007 for the verification of the effective junction area by using a graphene Josephson junction as a reference device.

[34] P. A. Rosenthal, M. R. Beasley, K. Char, M. S. Colclough, and G. Zaharchuk, Flux Focusing Effects in Planar ThinFilm Grain-Boundary Josephson Junctions, Appl. Phys. Lett. 59, 3482 (1991).

[35] C. W.J. Beenakker, Three "Universal" Mesoscopic Josephson Effects, Springer Ser. Solid-State Sci. 109, 235 (1992).

[36] T. D. Stanescu, J. D. Sau, R. M. Lutchyn, and S. Das Sarma, Proximity Effect at the SuperconductorTopological Insulator Interface, Phys. Rev. B 81, 241310(R) (2010).

[37] J. D. Sau, R. M. Lutchyn, S. Tewari, and S. Das Sarma, Robustness of Majorana Fermions in ProximityInduced Superconductors, Phys. Rev. B 82, 094522 (2010). 